\title{
ADORA1 is a diagnostic-related biomarker and correlated with immune infiltrates in papillary thyroid carcinoma
}

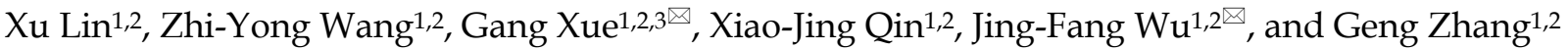 \\ 1. Zhangjiakou Key Laboratory of Thyroid Cancer Precision Diagnosis, Hebei North University, Zhangjiakou, 075000, China. \\ 2. Department of Histology and Embryology, Hebei North University, Zhangiiakou, 075000, China. \\ 3. Department of Otorhinolaryngology Head and Neck Surgery, Hebei North University, Zhangjiakou, 075000, China. \\ $\triangle$ Corresponding authors: Jing-fang Wu \& Gang Xue; E-mail: wjfxg@163.com; and, xgwjf@163.com, respectively. Jing-fang Wu, Department of histology and \\ embryology, Hebei North University, Zhangjiakou 075000, China. Telephone: +86-313- 4029346; Mobile: 086-18931316376; Fax: +86-313-4029289. \\ (0) The author(s). This is an open access article distributed under the terms of the Creative Commons Attribution License (https://creativecommons.org/licenses/by/4.0/). \\ See http://ivyspring.com/terms for full terms and conditions.
}

Received: 2020.07.16; Accepted: 2021.04.25; Published: 2021.05.13

\begin{abstract}
Background: Adenosine Al Receptor (ADORA1) is an adenosine receptor particularly relevant to the immunomodulatory process of malignant tumors. There are growing evidences that dysregulated overexpression of ADORAI can promote many types of tumorigenesis. However, the expression and prognostic value and mechanism of ADORA1 in thyroid papillary carcinoma have not been reported.

Methods: TCGA, ONCOMINE, UALCAN, cBioPortal, GeneMANIA, LinkedOmics, TIMER, GSCALite, TISIDB and EPIC tools were used in this study.

Results: ADORA1 was overexpressed in papillary thyroid carcinoma compared to paracancerous tissue. And ADORAI was positively correlated with lymph node metastasis as well as pathological stage in PTC. ADORAl had diagnostic and prognostic value for PTC. The functions of ADORAl co-expressed genes were mainly enriched in immune response, immune response-regulation signaling pathway, regulation of leukocyte activation and cancer-related pathways. Besides, ADORAl expression was significantly correlated with tumor-infiltrating cells and immune biomarkers in PTC. Finally, the high expression of ADORAl was sensitive to JW-55 drug.
\end{abstract}

Conclusion: ADORA1 is a diagnostic and a prognostic biomarker for PTC. The expression of ADORA1 is positively correlated with many immunoregulatory factors in PTC.

Key words: papillary thyroid carcinoma; ADORA1; immune infiltrates; biomarker

\section{Introduction}

Thyroid papillary carcinoma (PTC), originating from thyroid follicular epithelial cells, is a differentiated endocrine malignancy [1]. The incidence of PTC has been steadily increasing, and the incidence rate reached 6.20 per 100,000 in females and 1.80 per 100,000 in males on a world-wide scale [2]. Patients suffering from PTC with cervical lymph node metastases or distant metastases usually have a poor prognosis, and a small percentage of patients (about 10-15\%) may develop into a life-threatening recurrent disease [3-6]. Although the evolution of PTC is thought to be influenced by many factors such as genetic factors, hormone levels and ionizing radiation, the specific pathogenesis of PTC is still unclear, especially the underlying mechanism of immune regulation of specific genes [7-9]. Vigneri et al. noted that about half of PTC patients were accompanied by immune infiltration of macrophages and $\mathrm{T}$ lymphocytes [9]. And immunoregulatory targets such as PDL1, PDL2, PD1, LAG-3, TIM-3 were all aberrantly expressed in PTC [9].

Adenosine A1 Receptor (ADORA1) is an adenosine receptor particularly relevant to the immunomodulatory process of malignant tumors, with four family members (ADORA1, ADORA2a, ADORA2b and ADORA3) playing regulatory roles in vitro [10-12]. Adenosine has been reported to accumulate abnormally in the tumor microenvironment and binds to adenosine receptors to maintain the immune homeostasis in cancers. It has 
been well documented that abnormally overexpression of ADORA1 can facilitate the malignant progressions of colon cancer [13], kidney cancer [14], breast cancer [15], glioblastoma [16] and leukemia [17]. Programmed cell death protein 1 (PD-1) and its ligand (PD-L1) have been previously reported as immunotherapeutic targets for many solid cancers with significant efficacy [18-20]. Liu et al demonstrated that silencing ADORA1 expression in human melanoma cell lines significantly increased tumor PD-L1 levels and inhibited T cell-mediated cytotoxicity by conducting $\mathrm{T}$ cell-mediated cancer cell-killing assays [11]. These evidences all suggest that ADORA1 may mediate the immune microenvironment to influence cancer progression and the regulation of immunity. However, the specific mechanisms by which ADORA1 regulates tumor progression and immune cell infiltration in PTC are unclear.

Herein, we first analyzed the ADORA1 expression in PTC using the TCGA database, Oncomine database, and then tested whether ADORA1 expression in tissue microarrays was consistent with bioinformatics predictions using immunohistochemical staining experiments. ADORA1 gene alterations, functional enrichment analysis, protein-protein interaction networks, single nucleotide variation and ADORA1 associated regulatory factors (kinase, miRNA and transcription factor-target) in PTC were comprehensively evaluated. We analyzed the association between ADORA1 and tumor immune cell infiltration, and the relationship between ADORA1 and immune-related modulators using Tumor Immunoassay Resource (TIMER) and TISIDB tools, respectively. Finally, the relationships between differentially expressed ADORA1 and immune cells in PTC were analyzed using EPIC based on TCGA data.

\section{Materials and methods}

\section{Data preparation and cBioPortal}

Data concerning the ADORA1 mRNA expression profiles across the 512 TC samples and 18 normal tissue were downloaded from The Cancer Genome Atlas (TCGA) database. The OncoPrint of ADORA1and the relationships between the ADORA1 genetic variations and arm-level SCNA cluster assignment, extrathyroidal extension and disease stage were analyzed using the cBioPortal website, according to Papillary Thyroid Carcinoma (TCGA, Cell 2014) data [21]. Patients meeting the following criteria were excluded: patients had clinical information but no gene expression data.

\section{Tissue samples}

The PTC tissue microarrays were acquired from Shanghai Outdo Biotech CO., LTD. (NO. HThyPap120CS-01, Shanghai, China). Patients meeting the following criteria were excluded: (1) all patient samples in this tissue microarray were pathologically diagnosed as PTC; (2) all patient samples required no additional treatment. And the exclusion criteria were as follows to exclude patients with incomplete information on clinicopathological data.

\section{UALCAN analysis and Oncomine database analysis}

Both the UALCAN [22] and Oncomine [23] databases were online sites where you could acquire the expression, clinical stage, and survival status data of genes. In this study, we first used the UALCAN and Oncomine databases to analyze ADORA1 expression in PTC and paracancerous tissues. The expression of ADORA1 in PTC patients with different stage, gender, age, race and lymph node metastasis were then analyzed. P-value less than 0.05 was considered statistically significant.

\section{Kaplan Meier plotter analysis and LinkedOmics analysis}

The overall survival and relapse free information of ADORA1 were analyzed by Kaplan Meier plotter based on GEO and TCGA data [24]. LinkedOmics [25] was a comprehensive web application that could be used to analyze specific genes and their interconnections, functional enrichment analysis and certain regulatory factors in specific cancers. The interconnections, functional enrichment analysis (GO and KEGG analysis) and certain regulatory factors (transcriptional factors, microRNAs and protein kinases) of ADORA1 were analyzed by LinkedOmics.

\section{Immunohistochemistry}

Immunohistochemistry of ADORA1 was performed using a rabbit monoclonal anti-ADORA1 antibody (1:500 dilution, Bioss, bs-6649R).

Concrete procedure was referred to the previous study [26]. Briefly, $4 \mu \mathrm{m}$ PTC tissue microarray sections were dewaxed, hydrated and antigenrepaired before incubation with anti-ADORA1 primary antibody (1:500 dilution) at $4{ }^{\circ} \mathrm{C}$ overnight. 120-point PTC tissue chips were then washed with PBS and incubated with broad-spectrum secondary antibodies. Finally, the PTC tissue microarrays were photographed using an Olympus Biomicroscope. And the overall IHC score of ADORA1 in PTC and corresponding paracancerous tissue grading from 1 to 5 was assessed owing to the semi-quantitative immunoreactive score (IRS) scale of Remmele [27]. 
The percentage of tumor cells was scored as follows: 0 (0-5\% positive cells); 1 (6-25\% positive cells); 2 (26-50\% positive cells); 3 (51-75\% positive cells); and 4 (76-100\% positive cells). Staining intensity was scored as follows: 0 (no staining); 1-2 (weak staining); and $>2$ (strong staining).

\section{GeneMANIA analysis and GSCALite analysis}

GeneMANIA was an analytical tool to analyze the interconnection of gene clusters [28]. Interactions between ADORA1 and its top-four most significantly associated genes were analyzed. The GSCALite website was a multifunctional genomics site that could be used to analyze gene expression, methylation, single nucleotide variation, pathway activity and drug targets in our study with TCGA PTC sample [29]. The single nucleotide variation, drug targets and pathway activity of ADORA1, MYBPH, FAM178B, CHI3L1, METTL7B were analyzed using GSCALite tool. $\mathrm{p}<0.05$ was considered statistically significant.

\section{Immune-related analysis}

TIMER, an interactive web server, enabled online analysis of immune cells, immune genes and immunomodulatory factors in a variety of cancers using TCGA database data [30]. Analysis of ADORA1 expression in relation to biomarker levels of tumorinfiltrating immune cells using the "correlation" module. TISIDB was a web portal that integrates multiple heterogeneous data types on the interaction of tumor immunomodulatory factors [31]. In this experiment, we analyzed the regulatory factors and immune subtype that could be involved in ADORA1 immunoregulation in the PTC. EPIC could complete the estimation of immune cells in cancer samples based on its own algorithm by integrating gene expression in different cancer samples [32]. To assess the effect of ADORA1 expression on immune cells, we classified the 512 PTC samples from TCGA into the ADORA1 high expression group (top 256 cases) and the ADORA1 low expression group (low 256 cases). The EPIC application tool and GraphPad Prism 7 were used for immunoassay and results visualization, respectively. $\mathrm{p}<0.05$ was considered statistically significant.

\section{Results}

\section{Expression and prognostic value of ADORAI in PTC}

To illuminate the role of ADORA1 in papillary thyroid cancer, we first evaluated its expression, diagnostic value and prognostic value in patients with PTC. Data in the TCGA database and the Oncomine database revealed that mRNA expression of ADORA1 were significantly higher in papillary thyroid cancer tissues than that in normal tissues (Figure 1). Then, we analyzed the expression of ADORA1 with the tumor stage, patients' age, patients' race, patients' gender and nodal metastasis status for thyroid carcinoma. Regardless of tumor stage, race, gender, age and nodal metastasis status, ADORA1mRNA levels in PTC tissues were significantly higher than in normal thyroid tissues (Figure 2). The prognostic value of ADORA1 mRNA expression levels in thyroid cancer was conducted utilising Kaplan-Meier Plotter. The results revealed that high expression of ADORA1 $(\mathrm{HR}=2.87$, 95\% CI: 1.25-6.6, and $\mathrm{p}=0.0094)$ was associated with a worse disease free survival in the thyroid carcinoma patients while high expression of ADORA1 (HR=0.36, 95\% CI: 0.14-0.97, and $\mathrm{p}=0.035$ ) was associated with a longer overall survival (Figure 3). Besides, immunochemical staining experiment of PTC tissue microarrays (Shanghai cohort) revealed that ADORA1 was overexpressed in PTC tissues compared to paracancerous tissues and ROC curves were performed to assess the diagnostic role of ADORA1 in PTC (Figure 4). And ADORA1 expression was positively correlated with pathological stage $(p=0.00001, r=0.632)$ and lymph node metastasis $(\mathrm{p}=0.01, \mathrm{r}=0.64)$ in Shanghai cohort (Table 1). As shown in Figure 4, the AUC of ADORA1 in TCGA cohort was 0.9408 (95\% CI: 0.9209- 0.9606, p $<0.0001$ ) and the AUC of ADORA1 in Shanghai cohort was 0.9064 (95\% CI: 0.8546-0.9581, p <0.0001). Thus, ADORA1 can be considered as a potential diagnostic indicator of PTC.

Table 1. ADORAl expression and clinicopathological characteristics of 58 specimens in Shanghai corhort

\begin{tabular}{|c|c|c|c|c|c|c|}
\hline \multirow[t]{2}{*}{ Characteristics } & \multirow[t]{2}{*}{ Total } & \multicolumn{2}{|c|}{$\begin{array}{l}\text { ADORA1 } \\
\text { expression }\end{array}$} & \multirow[t]{2}{*}{$P$ value } & \multirow[t]{2}{*}{$\chi^{2}$ value } & \multirow{2}{*}{$\begin{array}{l}\text { Correlation } \\
\text { r value }\end{array}$} \\
\hline & & Low & High & & & \\
\hline Age (years) & & & & 0.074 & 3.194 & \\
\hline$<45$ & 24 & 9 & 15 & & & \\
\hline$\geq 45$ & 34 & 22 & 12 & & & \\
\hline Gender & & & & 0.5 & 3.587 & \\
\hline Female & 13 & 6 & 7 & & & \\
\hline Male & 45 & 25 & 20 & & & \\
\hline Pathological stage & & & & 0.00001 & 23.161 & 0.632 \\
\hline Low $(\mathrm{I}+\mathrm{II})$ & 37 & 28 & 9 & & & \\
\hline High (III +IV) & 21 & 3 & 18 & & & \\
\hline Lymph node metastasis & & & & 0.01 & 9.757 & 0.640 \\
\hline Negative & 32 & 22 & 10 & & & \\
\hline Positive & 26 & 9 & 17 & & & \\
\hline
\end{tabular}

\section{Genomic alterations and enrichment analysis of ADORAl correlated genes in PTC}

Due to the significance of ADORA1 in PTC, the types and frequency of ADORA1alterations in PTC were analyzed by cBioPortal tool according to DNA sequencing data from PTC patients. ADORA1 was 
altered in 22 of 338 (6\%) PTC patients. These alterations involved mRNA up-regulation in 18 cases $(4.6 \%)$ and amplification in 4 cases (1.4\%) (TCGA, Cell 2014) (Figure 5A). Besides, the frequency distribution of ADORA1 CNV patients in arm-level SCNA cluster assignment, different stage and extrathyroidal extension were presented in Figure 5B-5D, suggesting the early-event and disease progression of ADORA1 CNV alteration in PTC.

\section{A

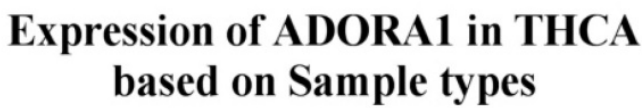

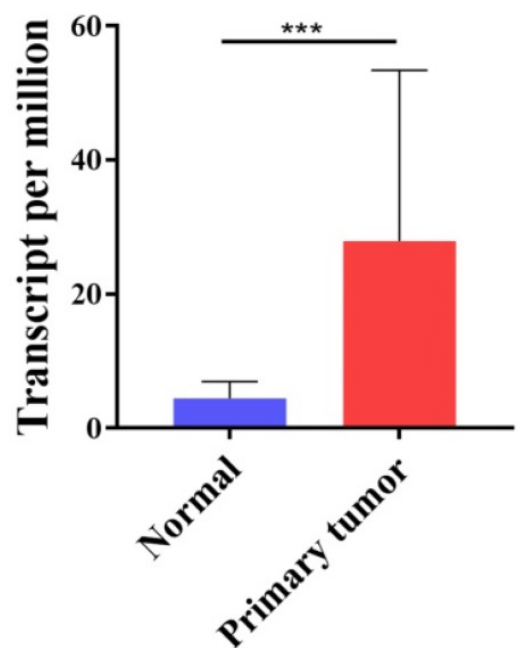

B Thyroid Gland Papillary Carcinoma vs. Normal

He Thyroid Statistics

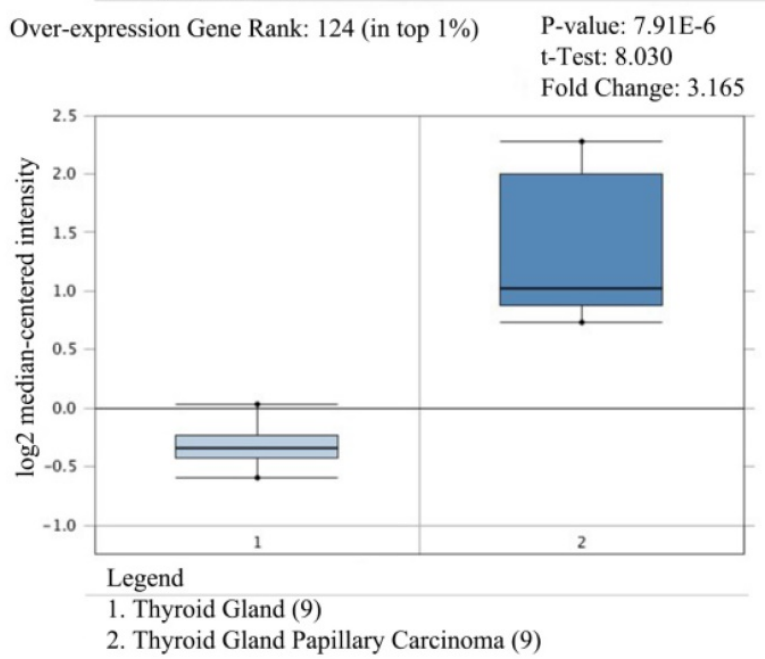

Figure 1. The transcription levels of ADORA1 in thyroid cancer. Compared with normal samples, ADORA1 mRNA was overexpressed in PTC (A) UALCAN database and (B) ONCOMINE database. $* * * * 0.001$.

Expression of ADORA1 in THCA based on individual cancer stages

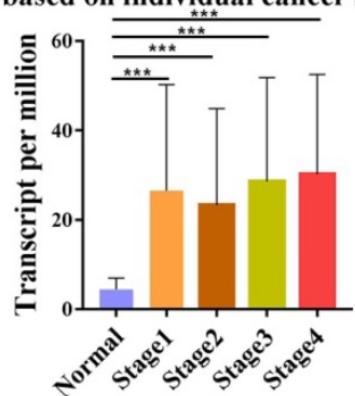

Expression of ADORA1 in THCA based on patient's race

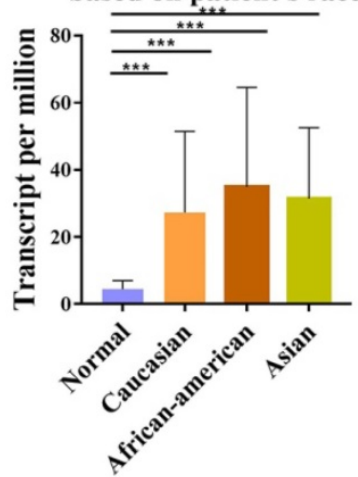

Expression of ADORA1 in THCA based on patient's age

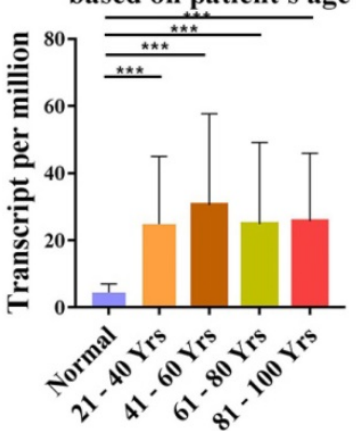

Expression of ADORA1 in THCA based on patient's gender

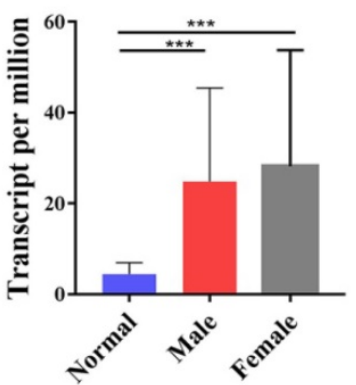

Figure 2. Correlation between ADORAl expression and tumor stage in TC patients (UALCAN). Compared with normal tissues, ADORAl expression was positively correlated with the nodal metasis, clinical stage. ${ }^{*} p<0.05,{ }^{*} p<0.01$, $* * * *<<0.001$. 

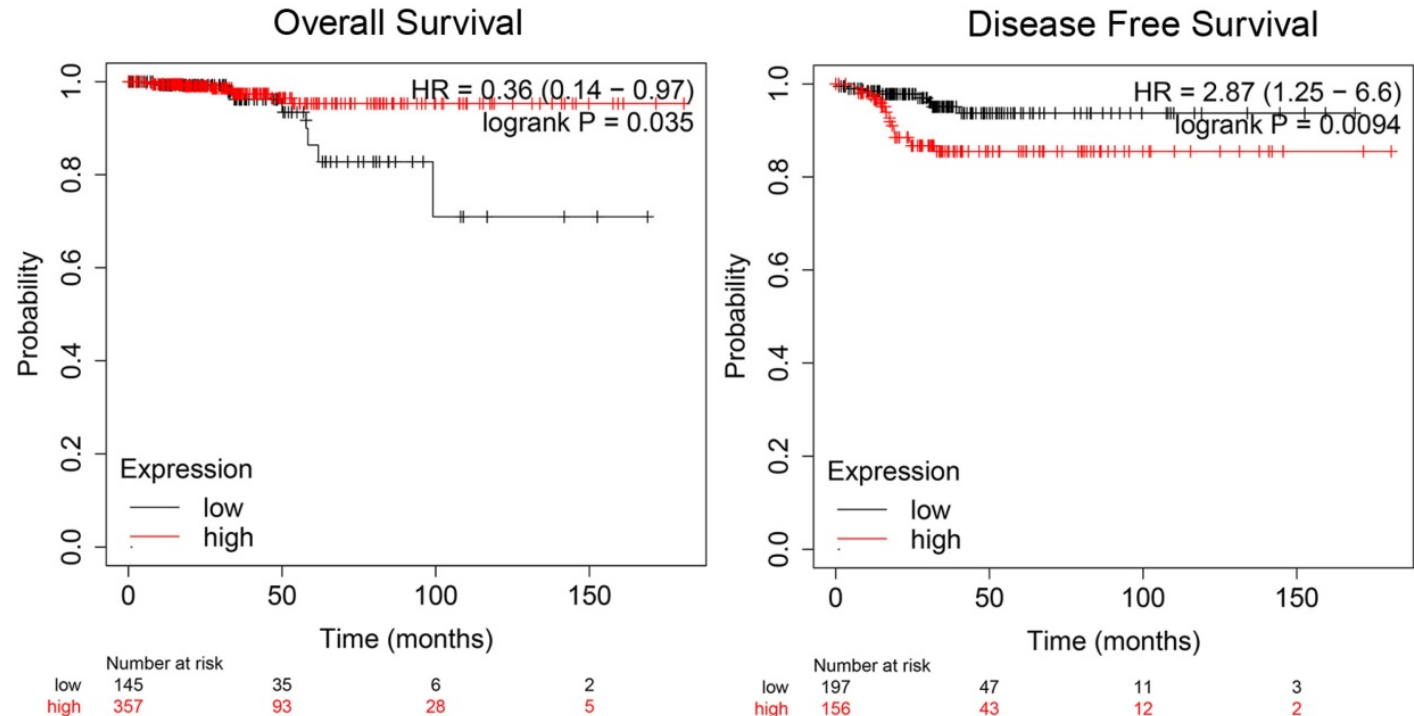

Figure 3. Prognostic value of mRNA expression of ADORA1 in thyroid cancer patients (Kaplan-Meier Plotter).
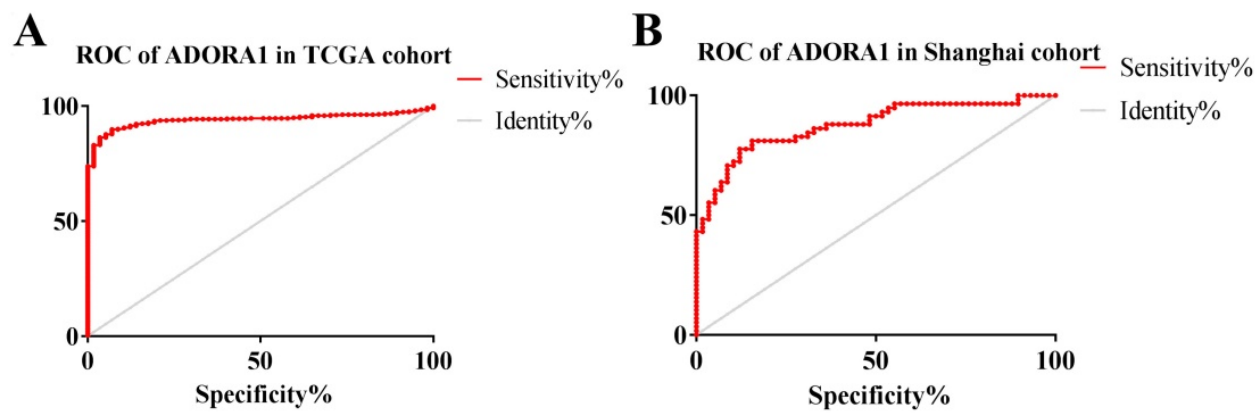

C

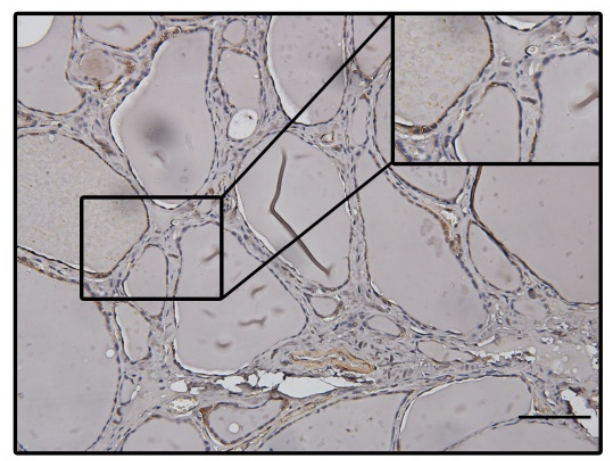

Normal tissue
D

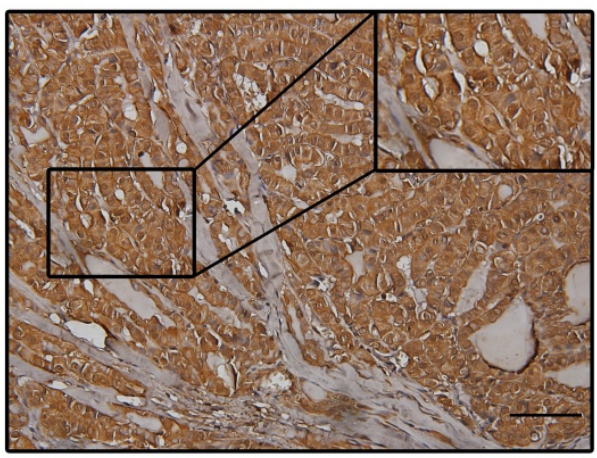

PTC tissue

Figure 4. ROC curves and immunohistochemistry results of ADORA1. (A) AUC of ADORA1 in TCGA cohort was 0.9408 (95\% Cl: $0.9209-0.9606$ ), P <0.0001. (B) AUC of ADORAl in Shanghai cohort was 0.9064 (95\% Cl: 0.8546-0.9581), p <0.0001. (C-D) ADORA1 proteins were higher in PTC tissues, compared to tumor-adjacent tissues, Scale bar: $20 \mu \mathrm{m}$.

To gain insight into the biological significance and function of ADORA1 in PTC, multiple modules of LinkedOmics were used to examine ADORA1 co-expression patterns, functional enrichment analysis and regulators in the PTC cohort. As shown in Figure 6A, 5713 genes (dark red dots) were shown positive correlations with ADORA1, whereas 7050 genes (dark green dots) were shown negative correlations with ADORA1 (false discovery rate, FDR $<0.05)$. In addition, the top 50 important genes positively and negatively associated with ADORA1 in the PTC were presented in Figure $6 \mathrm{~B}$ and $6 \mathrm{C}$, respectively. Moreover, ADORA1 and the top 4 significant genes, including $\mathrm{MYBPH}$ (cor=0.0.71, $\mathrm{p}=4.86 \mathrm{e}-81), \quad$ FAM178B (cor $=0.69, \quad \mathrm{p}=1.22 \mathrm{e}-72)$, CHI3L1 (cor $=0.68, \mathrm{p}=1.74 \mathrm{e}-70$ ) and METTL7B (cor $=$ $0.67, \mathrm{p}=2.68 \mathrm{e}-680)$, positively correlated with ADORA1 in PTC, were considered as the hub genes (Supplementary Figure 1). 
A

ADORA1 $6 \%$

mRNA Expression z-Scores (RNA Seq V2 RSEM)

ADORA1

\begin{tabular}{l||l||l} 
Genetic Alteration & Amplification mRNA High & No alterations
\end{tabular}

Expression Heatmap

$-3$

3

$\mathbf{B}$

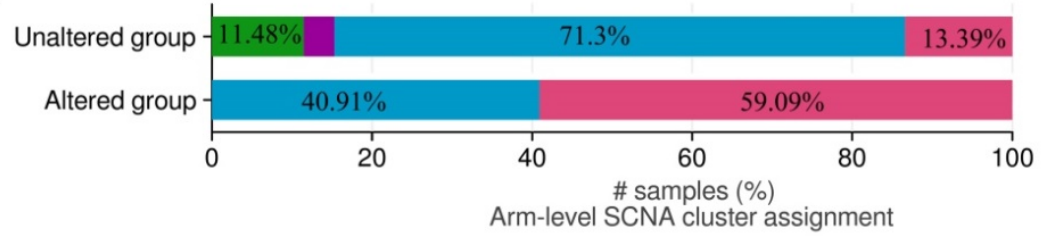

- 22q Many SCNA घuiet - SomeSCNA

C

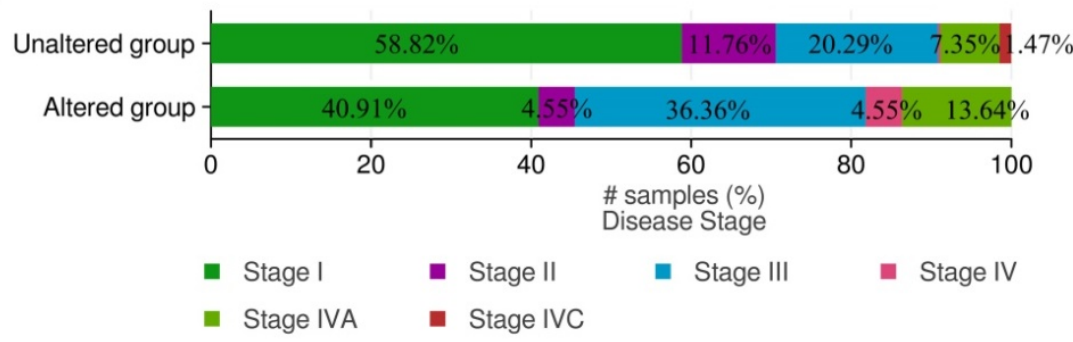

D

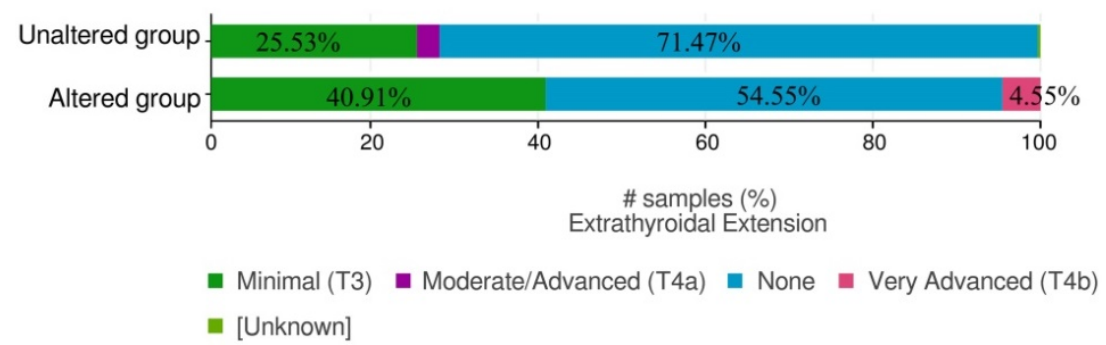

Figure 5. ADORAl genomic alterations in PTC. (A) OncoPrint of ADORAl alterations in PTC cohort. (B) Distribution of ADORAI CNV frequency in arm-level SCNA cluster assignment. (C) Distribution of ADORAI CNV frequency in different stages. (D) Distribution of ADORAI CNV frequency in different extrathyroidal extension.

Significant Gene Ontology (GO) term annotation showed that ADORA1 correlated genes mainly participated in adaptive immune response, immune response-regulation signaling pathway, regulation of leukocyte activation, granulocyte activation, cilium organization, mitochondrial gene expression, response to virus, microtubule-based movement and generation of precursor metabolites and energy (Figure 6D). The KEGG pathway indicated that ADORA1 and its related genes were primarily associated with cell adhesion molecules (CAMs), natural killer cell-mediated cytotoxicity, Th17 cell differentiation, epstein-barr virus infection, bacterial invasion of epithelial cells, phagosome, influenza A, complement and coagulation cascades and valine, leucine and isoleucine degradation (Figure 6E).

\section{Kinase, miRNA or transcription factor targets of ADORAl in PTC}

To further explore the regulators of ADORA1 in PTC, we performed an analysis of kinases, miRNAs and transcription factors of ADORA1 gene (Table 2). For kinase networks of ADORA1, only one kinase target of ADORA1 was identified (Kinase_STK11) according to the LinkedOmics database. To probe the function of kinase STK11 in more details, we next constructed PPI networks of STK11. And the results showed that these genes were primarily involved in the regulation of insulin receptor signaling pathway, cell cycle arrest, negative regulation of cell cycle, cellular response to peptide hormone stimulus, protein serine/threonine kinase activity, protein kinase activator activity (Supplementary Figure 2). 
For miRNAs, MIR-221 and MIR-222 were enriched by GSEA of ADORA1 co-expressed genes. Interestingly, the functions of the genes of MIR-222 network were mainly involved in protein insertion into mitochondrial membrane involved in apoptotic signaling pathway, positive regulation of protein insertion into mitochondrial membrane involved in apoptotic signaling pathway, regulation of chromatin silencing, regulation of chromosome organization, negative regulation of gene expression, epigenetics, protein insertion into mitochondrial membrane, regulation of protein insertion into mitochondrial membrane involved in apoptotic signaling pathway (Supplementary Figure 3). Moreover, the transcripttion factors (GGGNNTTTCC_V\$NFKB_Q6_01, V\$AP1_Q4_01, V\$AP1_C, V\$AP1_Q4, V\$PEA3_Q6, V\$NFKB_Q6, V\$NFKAPPAB_01， TGASTMAGC V\$NFE2_01， TGANTCA_V\$AP1_C, V\$STAT_01, V\$AP1_Q6, V\$TEF1_Q6, V\$BACH1_01, V\$PAX5_02, V\$AP1FJ_Q2, V\$AP1_Q2_01，V\$AP1_Q2, TTCYN RGAA_V\$STAT5B_01, V\$BACH2_01, V\$NFKB_Q6_ 01， RYTTCCTG_V\$ETS2_B, V\$SREBP1_Q6 and V\$ELF1_Q6) of ADORA1 were shown in Table 2. And the functions of these transcription factors were primarily enriched in plasma lipoprotein particle assembly, protein-lipid complex assembly, macrophage-derived foam cell differentiation, foam cell differentiation, protein-lipid complex subunit organization, sequence-specific cDNA binding, regulation of plasma lipoprotein particle levels (Supplementary Figure 4).

Table 2. The Kinase, miRNA and transcription factor-target networks of ADORAl in Thyroid carcinoma (LinkedOmics)

\begin{tabular}{llll}
\hline $\begin{array}{l}\text { Enriched } \\
\text { Category }\end{array}$ & Geneset & LeadingEdgeNum FDR \\
\hline Kinase Target & Kinase_STK11 & 13 & 0.02804 \\
miRNA Target & ATGTAGC,MIR-221,MIR-222 & 45 & 0.045 \\
Transcription & GGGNNTTTCC_V\$NFKB_Q6_01 & 50 & 0 \\
Factor Target & V\$AP1_Q4_01 & 71 & 0.0045693 \\
& V\$AP1_C & 68 & 0.0049847 \\
& V\$AP1_Q4 & 66 & 0.0054001 \\
& V\$PEA3_Q6 & 91 & 0.0055385 \\
& V\$NFKB_Q6 & 89 & 0.0059816 \\
& V\$NFKAPPAB_01 & 70 & 0.0061715 \\
& TGASTMAGC_V\$NFE2_01 & 55 & 0.0074015 \\
& TGANTCA_V\$AP1_C & 243 & 0.0076155 \\
& V\$STAT_01 & 55 & 0.0078093 \\
& V\$AP1_Q6 & 63 & 0.0081232 \\
& V\$TEF1_Q6 & 51 & 0.00818 \\
& V\$BACH1_01 & 64 & 0.0083078 \\
& V\$PAX5_02 & 5 & 0.0087825 \\
V\$AP1FJ_Q2 & 61 & 0.0089724 \\
V\$AP1_Q2_01 & 67 & 0.010263 \\
V\$AP1_Q2 & 58 & 0.010904 \\
TTCYNRGAA_V\$STAT5B_01 & 98 & 0.013817 \\
V\$BACH2_01 & 66 & 0.014308 \\
V\$NFKB_Q6_01 & 64 & 0.014539 \\
RYTTCCTG_V\$ETS2_B & 295 & 0.018277 \\
V\$SREBP1_Q6 & 61 & 0.022198 \\
V\$ELF1_Q6 & 83 & 0.022431 \\
\hline
\end{tabular}

The correlation between ADORAl expression and immune biomarkers of ADORA1 in PTC

\section{It has been previously documented that} ADORA1 may mediate the immune microenvironment to influence cancer progression and immune regulation [18-20]. Thus, the correlations of ADORA1 expression in PTC with immune cells and biomarkers were investigated using the TIMER and TISIDB tools. As shown in Figure 7A, ADORA1 expression in the PTC was positively associated with $B$ cell (Cor $=0.282, \quad \mathrm{P}=2.92 \mathrm{e}-10), \mathrm{CD} 8+\mathrm{T}$ cells (Cor $=0.116, \mathrm{P}=1.04 \mathrm{e}-02), \mathrm{CD} 4+\mathrm{T}$ cells $(\mathrm{Cor}=0.105, \mathrm{P}=$ 2.08e-02), Macrophage (Cor $=0.14, \mathrm{P}=2.00 \mathrm{e}-03)$, Neutrphils (Cor $=0.251, \mathrm{P}=1.82 \mathrm{e}-08)$ and Dendritic cells $($ Cor $=0.308, \mathrm{P}=3.96 \mathrm{e}-12)$. Besides, we performed an analysis of the association between ADORA1 expression and immunomodulators using the TISIDB database. Figure 7B demonstrated correlations between ADORA1 expression and immune inhibitors. The immune inhibitors showed strong correlations with ADORA1 expression including CTLA4 (Spearman: $\rho=0.292, \quad P=2.28 \mathrm{e}-11$ ), TGFBR1 (Spearman: $\rho=0.427, \quad \mathrm{P}<$ 2.2e-16), HAVCR2 (Spearman: $\rho=0.275, P=3.31 \mathrm{e}-10$ ) and PDCD1LG2 (Spearman: $\rho=0.328, P=4.39 \mathrm{e}-14$ ) in PTC (Figure 7B-7C). For immunostimulators, ADORA1 expression was positively correlated with CD276 (Spearman: $\rho=$ 0.48, P < 2.2e-16), KLRC1 (Spearman: $\rho=0.385, \mathrm{P}<$ 2.2e-16), TMEM173 (Spearman: $\rho=0.417, \mathrm{P}<2.2 \mathrm{e}-16$ ) and TNFRSF18 (Spearman: $\rho=0.445, \mathrm{P}<2.2 \mathrm{e}-16$ ) in PTC (Figure 7D-7E). Figure 7F-7G revealed correlations between ADORA1 expression and MHC molecules. There were positive correlations between ADORA1 expression and HLA-A (Spearman: $\rho=$ 0.479, P < 2.2e-16), HLA-DMA (Spearman: $\rho=0.449$, P $<$ 2.2e-16), HLA-DQA1 (Spearman: $\rho=0.358, \mathrm{P}=$ 7.3e-17) and TAP1 (Spearman: $\rho=0.421, \mathrm{P}<2.2 \mathrm{e}-16$ ) in PTC. In addition, ADORA1 expression was related to immune subtypes in PTC including wound healing, IFN-gamma dominant, inflammatory, lymphocyte depleted, and TGF-b dominant (Figure 7H). To understand the relationships between ADORA1 expression and immune cell markers in more detail, the relationship between ADORA1 expression and many of these immune markers were analyzed. As shown in Table 3, remarkable correlations were found between ADORA1 expression and the expression of CD2, CD115, IRF5, CD11b, HLA-DPB1, HLA-DQB1, HLA-DRA, HLA-DPA1, BDCA-1, STAT4, STAT6, STAT5A and FOXP3. Finally, the relationships between different ADORA1 expression and immune cells in PTC patients were analyzed using the EPIC application. The results showed that $\mathrm{B}$ cells $(\mathrm{P}=$ $0.018)$, CAFs $(P=0.0039), C D 8 T$ cells $(P<0.0001)$, Endothelial cells $(\mathrm{P}=0.0006)$, Macrophage cells ( $\mathrm{P}$ 
$=0.0018$ ) were main immune cells affected by different ADORA1 expression (Figure 8).

The genetic variation, interrelationship, cancer pathway and drug susceptibility analysis of 5 hub genes
ADORA1 and the top 4 significant genes, including MYBPH, FAM178B, CHI3L1 and METTL7B were selected as the hub genes for genetic variation, interrelationship, cancer pathway and drug susceptibility analysis. For genetic variation analysis, waterfall plot showed the mutation distribution and

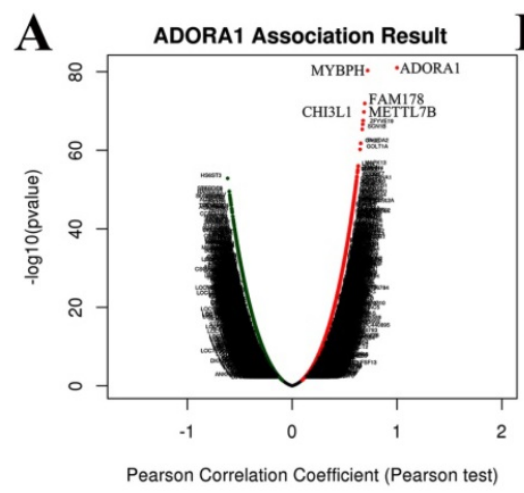

D

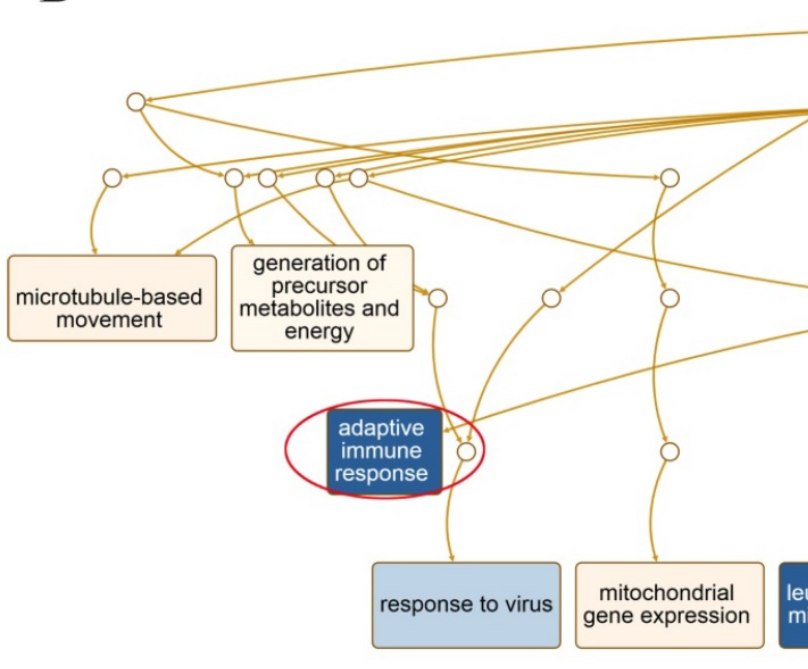

$\mathbf{E}$

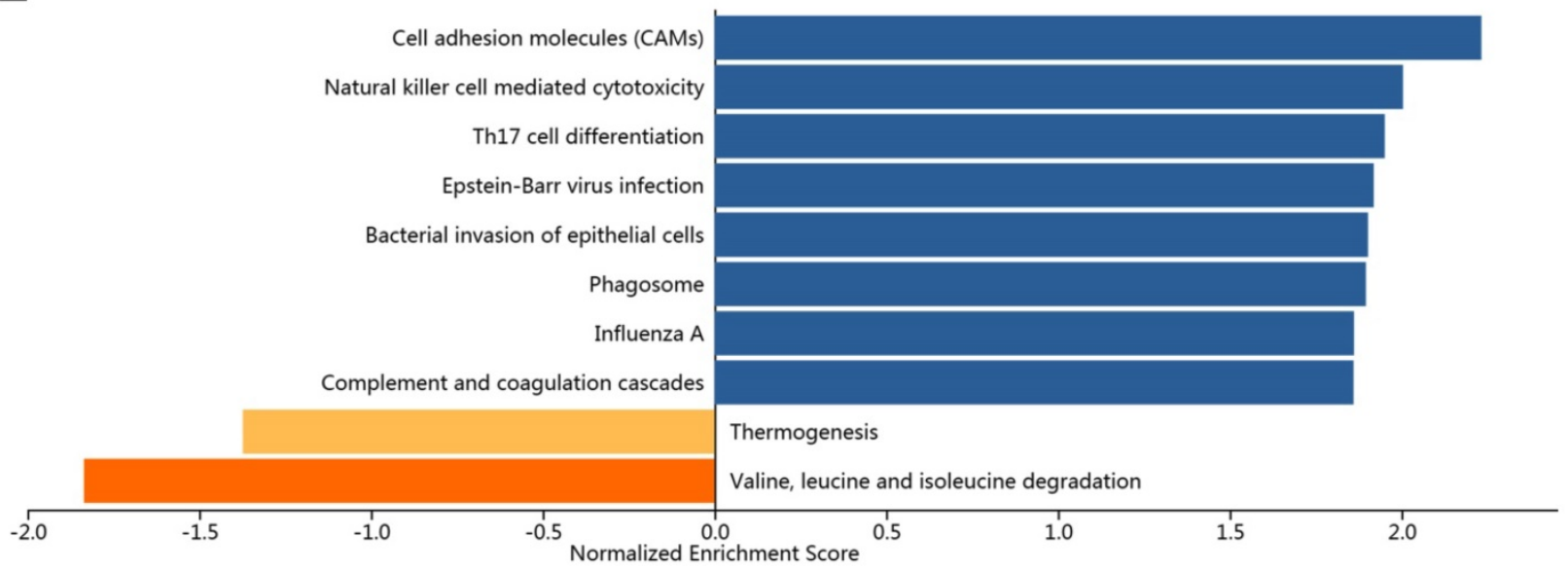

Figure 6. ADORA1 co-expression genes in PTC (LinkedOmics). (A) The genes positively and negatively correlated with ADORA1 in PTC. (B) The top-50 gene positively correlated with ADORA1 in PTC in heat maps. (C) The top-50 gene negatively correlated with ADORA1 in PTC in heat maps. (D) Biological process analyse and KEGG pathways of ADORA1 in TCGA cohort (E).

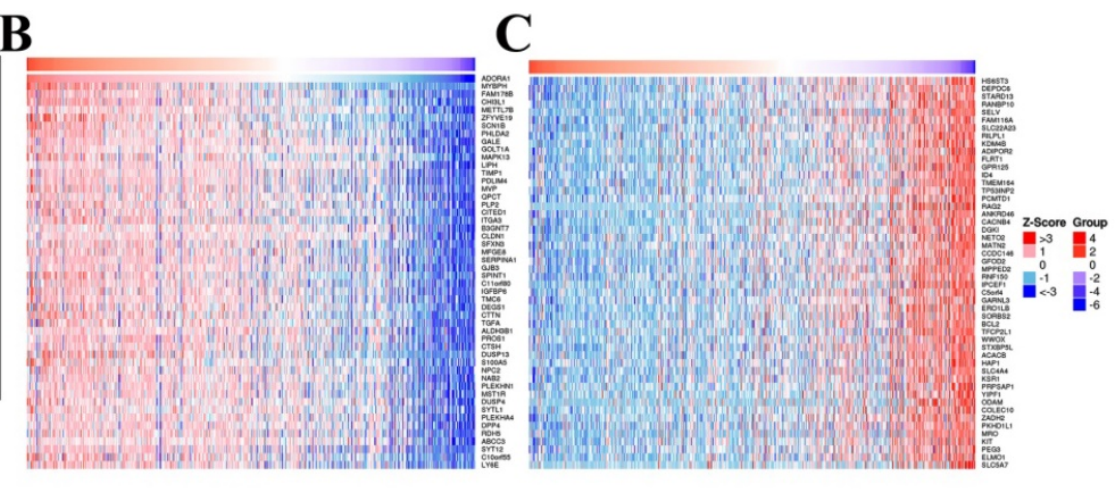

GO_BP

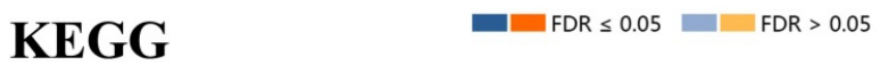


SNV classification types of 5 hub genes (Figure 9). And potential interactions between these 5 genes were displayed using GeneMANIA analysis (Figure 10A). Then, we used the GSCALite tool to analyze the potential roles of these 5 genes in the classical cancer pathways. The results showed that these five genes, especially ADORA1, could activate EMT, Hormone ER, PI3K/AKT, RAS/MAPK, TSC/mTOR pathways, and inhibit RTK, Hormone AR, DNA Damage Response, Cell Cycle, Apoptosis pathways to exert regulatory effects in cancer process (Figure 10B). For drug susceptibility analysis, through the GSCALite website we analyzed the relationship between ADORA1 expression and multiple drug sensitivities according to Therapeutics Response Portal (CTRP). The results showed that high expression of METTL78B was resistant to 41 drugs or small molecules. And the high expression of ADORA1 was also resistant to 46 drugs or small molecules. Besides, we found that high expression of ADORA1 was sensitive to JW-55 drugs (Figure 11). Therefore, this provided additional targets for PTC immunotherapy.

\section{Discussion}

Adenosine was well recognized as a potent modulator of both innate and acquired immunity [33]. To date, there were a total of four types of adenosine G-protein-coupled receptors, which were ADORA1, ADORA2a, ADORA2b and ADORA3 [34]. Hasko et al. noted that ADORA1 not only had the highest affinity for adenosine, but was also abundantly expressed in immune cells [35]. Moreover, a growing number of reports indicated that ADORA1 could influence the development, progression and metastasis of various kinds of cancers by mediating immune processes. However, the function of ADORA1 in PTC remains unclear. In other words, our study was the first one to investigate the roles of ADORA1 in PTC.

A

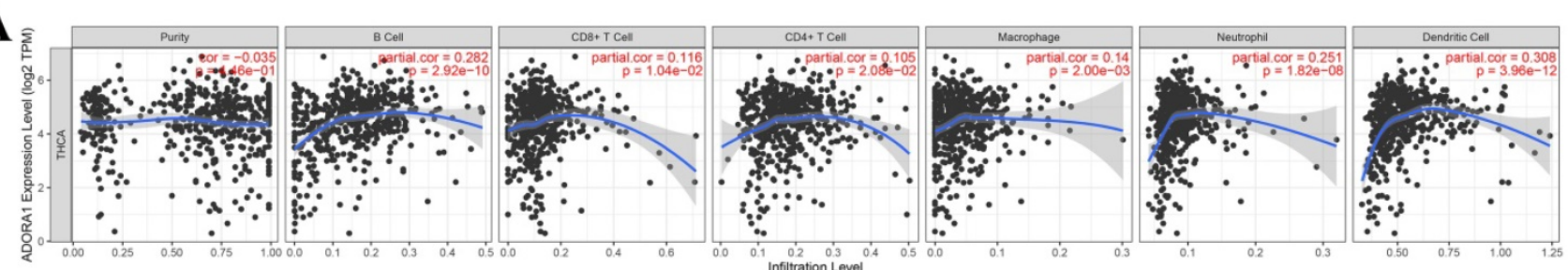

B

C

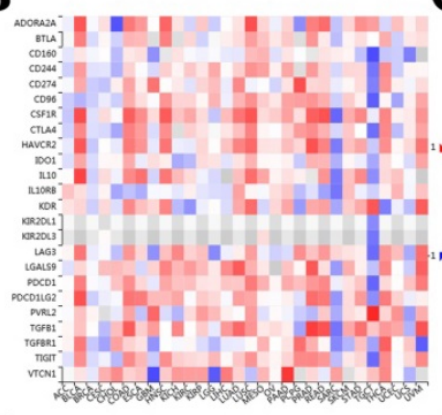

$\mathbf{F}$
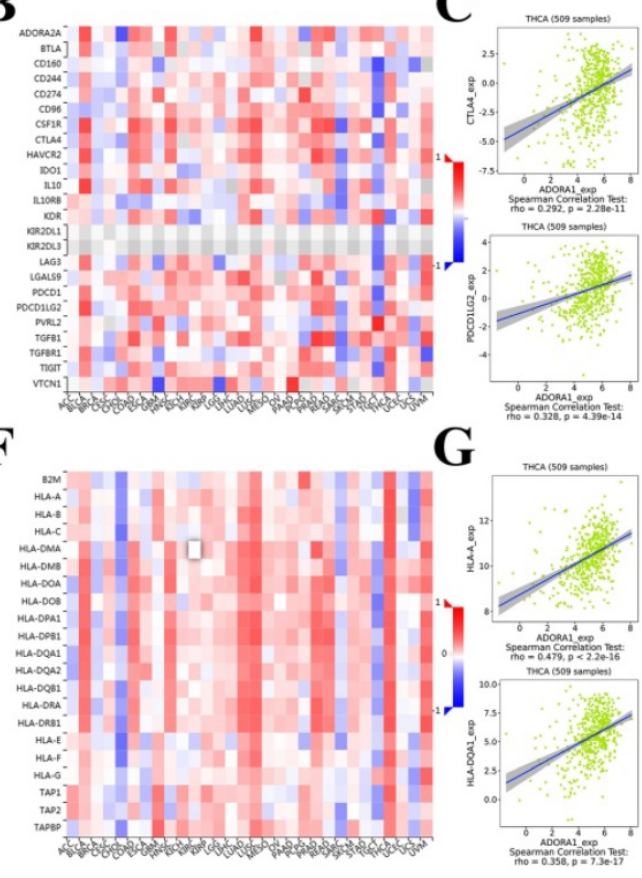
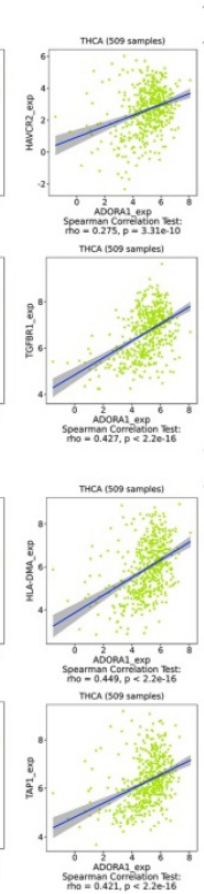

D

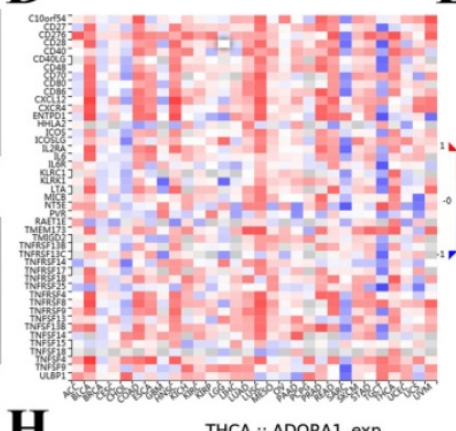

THCA :: ADORA1_exp

$\mathbf{E}$
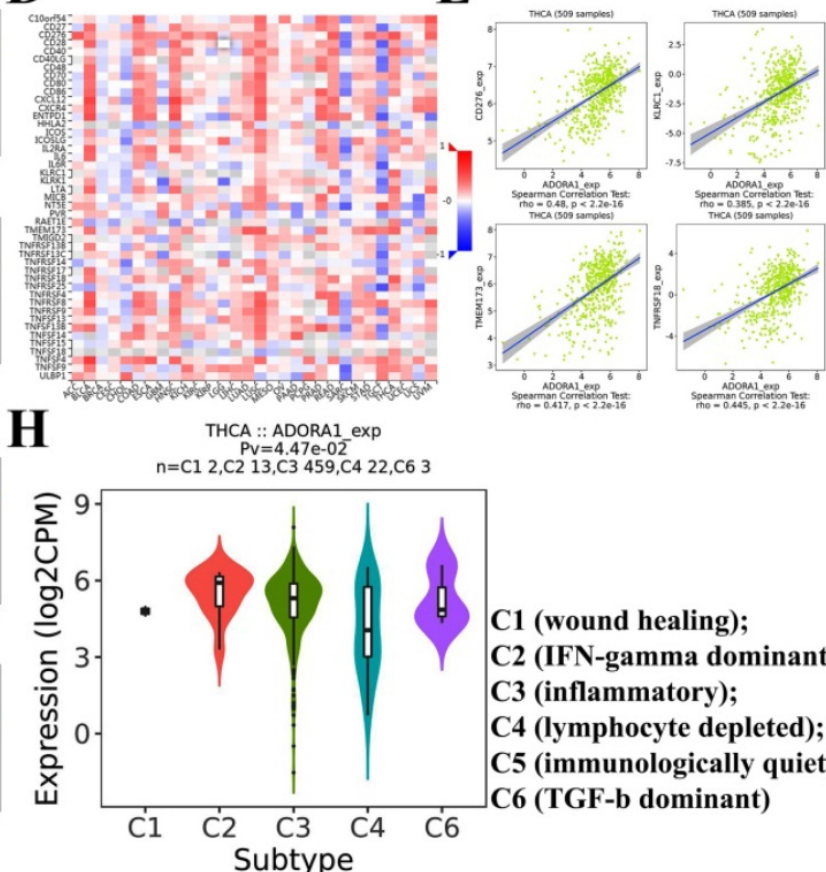

C1 (wound healing);

C2 (IFN-gamma dominant); C3 (inflammatory); C4 (lymphocyte depleted); C5 (immunologically quiet); C6 (TGF-b dominant)

Figure 7. Correlation of ADORAl expression with immune cell and immunomodulators in thyroid cancer. (A) ADORA1 expression in the PTC were positively associated with $B$ cell $(C o r=0.282, P=2.92 e-10), C D 8+T$ cells $(C o r=0.116, P=1.04 e-02), C D 4+T$ cells $($ Cor $=0.105, P=2.08 e-02)$, Macrophage $(C o r=0.14, P=2.00 e-03), N e u t r p h i l s(C o r=$ $0.251, P=1.82 \mathrm{e}-08$ ) and Dendritic cells $($ Cor $=0.308, P=3.96 \mathrm{e}-12)$. (B) Relations between the immunoinhibitors and ADORAl expression. (C) 4 immunoinhibitors were correlated with ADORAl expression. (D) Relations between the immunostimulators and ADORAl expression. (E) 4 immunostimulators were correlated with ADORA1 expression. (F) Relations between the MHC molecules and ADORA1 expression. (G) 4 MHC molecules were correlated with ADORA1 expression. (H) ADORA1 expression was related to immune subtypes in PTC including wound healing, IFN-gamma dominant, inflammatory, lymphocyte depleted, and TGF-b dominant. 

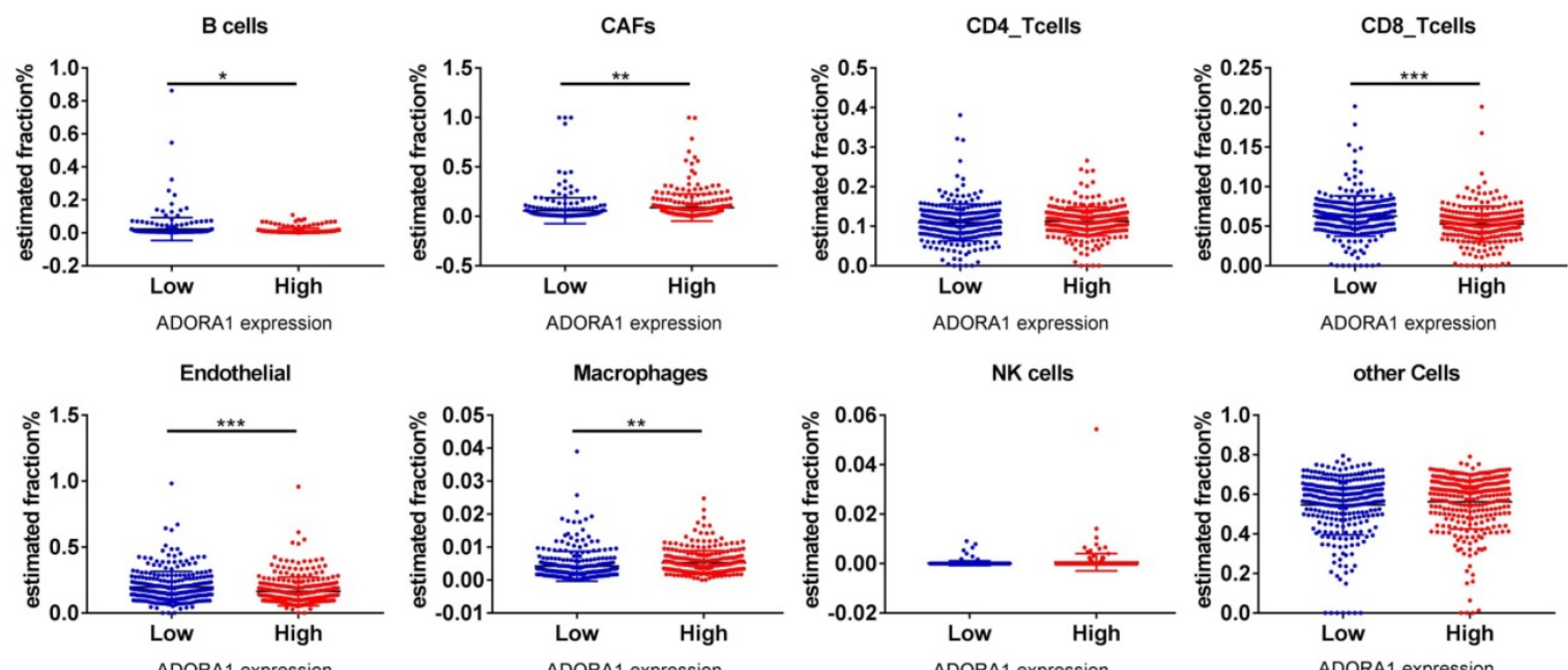

NK cells

other Cells
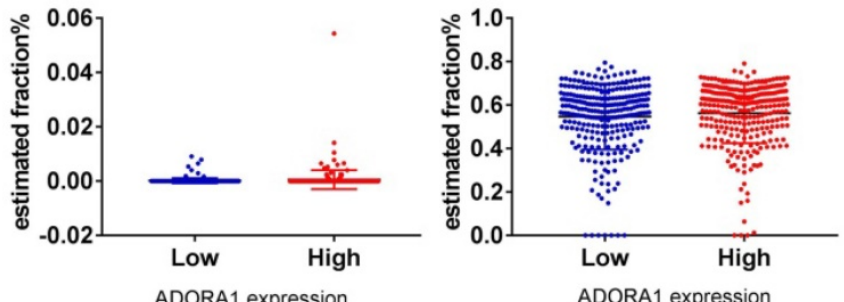

Figure 8. Different immune cells expression in ADORA1 low group and ADORA1 high group analyzing by online tools EPIC. *P $<0.05$, $* * P<0.01, * * * P<0.001$.

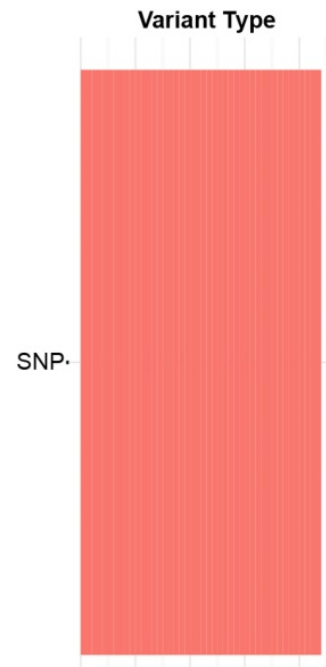

$\begin{array}{lllll}0 & 10 & 20 & 30 & 40\end{array}$ Variants per sample

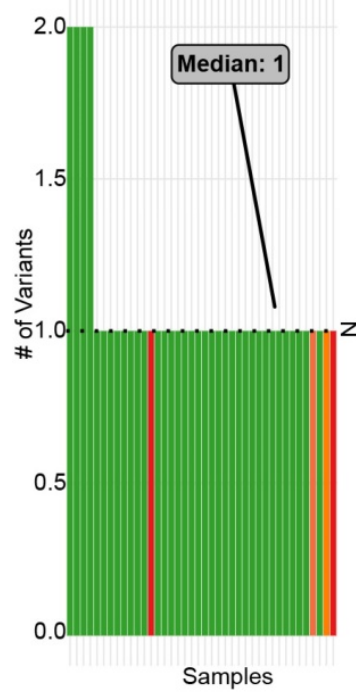

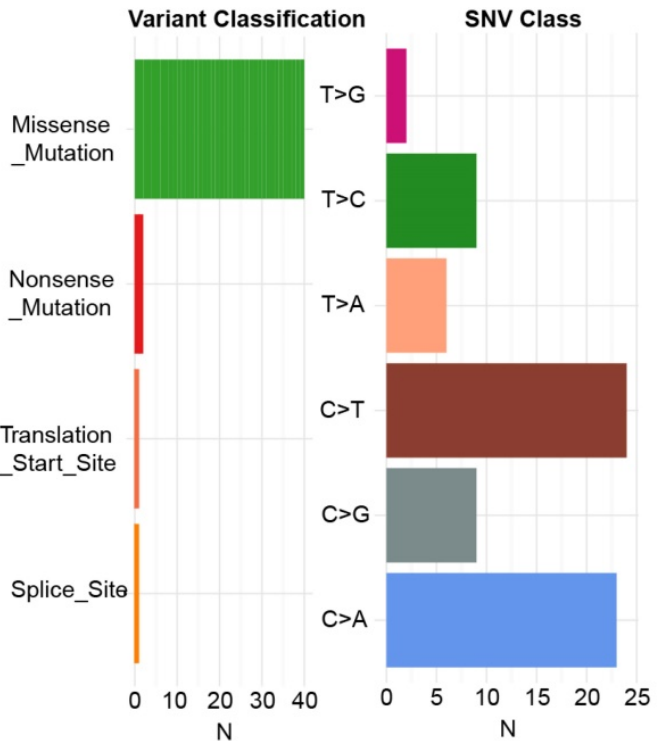

Variant Classification Summary Frequently Mutated Genes

6
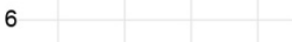

ADORA1

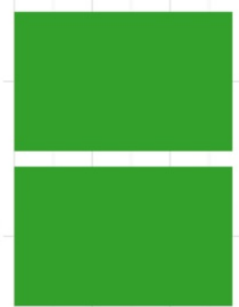

CHI3L1

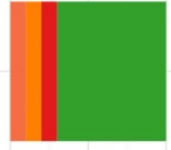

METTL7B

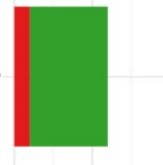

Variant_Classification $\quad 0 \quad 5 \quad 10$

Figure 9. The single nucleotide variation analysis of ADORA1, MYBPH, FAM178B, CHI3L1 and METTL7B. 
Table 3. Correlation analysis between ADORAl and gene biomarkers of immune cells in THCA (TIMER)

\begin{tabular}{|c|c|c|c|c|c|}
\hline \multirow{2}{*}{$\begin{array}{l}\text { Immune } \\
\text { cells }\end{array}$} & \multirow[t]{2}{*}{ Biomarkers } & \multicolumn{2}{|l|}{ None } & \multicolumn{2}{|l|}{ Purity } \\
\hline & & Cor & P-value & Cor & P-value \\
\hline \multirow[t]{2}{*}{ CD8+ T cell } & CD8A & 0.134 & $2.5 e-03$ & 0.134 & $3.11 \mathrm{e}-03$ \\
\hline & CD8B & 0.136 & $2.07 \mathrm{e}-03$ & 0.136 & $2.66 \mathrm{e}-03$ \\
\hline \multirow{3}{*}{$\begin{array}{l}\text { T cell } \\
\text { (general) }\end{array}$} & CD3D & 0.283 & $9.45 \mathrm{e}-11$ & 0.283 & $1.83 \mathrm{e}-10$ \\
\hline & CD3E & 0.278 & $2.19 \mathrm{e}-10$ & 0.275 & $6.27 \mathrm{e}-10$ \\
\hline & $\mathrm{CD} 2$ & 0.31 & $8.97 \mathrm{e}-13$ & 0.309 & $3.19 \mathrm{e}-12$ \\
\hline \multirow[t]{2}{*}{ B cell } & CD19 & 0.107 & $1.61 \mathrm{e}-02$ & 0.101 & $2.63 e-02$ \\
\hline & CD79A & 0.153 & $5.54 \mathrm{e}-04$ & 0.145 & $1.34 \mathrm{e}-03$ \\
\hline \multirow[t]{2}{*}{ Monocyte } & CD86 & 0.281 & $1.35 \mathrm{e}-10$ & 0.278 & $4.20 \mathrm{e}-10$ \\
\hline & CD115 (CSF1R) & 0.316 & $3.93 e-13$ & 0.316 & $8.16 \mathrm{e}-13$ \\
\hline \multirow[t]{3}{*}{ TAM } & CCL2 & 0.231 & $1.54 \mathrm{e}-07$ & 0.235 & $1.52 \mathrm{e}-07$ \\
\hline & CD68 & 0.278 & $1.67 \mathrm{e}-10$ & 0.276 & $5.20 \mathrm{e}-10$ \\
\hline & IL10 & 0.106 & $1.63 \mathrm{e}-02$ & 0.106 & $1.88 \mathrm{e}-02$ \\
\hline \multirow{3}{*}{$\begin{array}{l}\text { M1 } \\
\text { Macrophage }\end{array}$} & INOS (NOS2) & -0.073 & $9.82 \mathrm{e}-02$ & -0.068 & $1.33 \mathrm{e}-01$ \\
\hline & IRF5 & 0.363 & $2.61 \mathrm{e}-17$ & 0.366 & $6.94 \mathrm{e}-17$ \\
\hline & COX2(PTGS2) & 0.284 & $7.81 \mathrm{e}-11$ & 0.278 & $4.18 \mathrm{e}-10$ \\
\hline M2 & CD163 & 0.162 & $2.49 \mathrm{e}-04$ & 0.157 & $5.21 \mathrm{e}-04$ \\
\hline \multirow[t]{2}{*}{ Macrophage } & VSIG4 & 0.239 & $5 e-08$ & 0.241 & $6.93 \mathrm{e}-08$ \\
\hline & MS4A4A & 0.185 & $2.76 \mathrm{e}-05$ & 0.179 & 7.11e-05 \\
\hline \multirow[t]{3}{*}{ Neutrophils } & CD66b (CEACAM8) & 0.186 & $2.32 \mathrm{e}-05$ & 0.195 & $1.42 \mathrm{e}-05$ \\
\hline & CD11b (ITGAM) & 0.374 & $2.26 \mathrm{e}-18$ & 0.376 & 7.41e-18 \\
\hline & CCR7 & 0.189 & $1.89 \mathrm{e}-05$ & 0.187 & $3.15 \mathrm{e}-05$ \\
\hline \multirow{7}{*}{$\begin{array}{l}\text { Natural } \\
\text { killer cell }\end{array}$} & KIR2DL1 & -0.074 & $9.49 \mathrm{e}-02$ & -0.065 & $1.49 \mathrm{e}-01$ \\
\hline & KIR2DL3 & 0.11 & $1.27 \mathrm{e}-02$ & 0.114 & $1.14 \mathrm{e}-02$ \\
\hline & KIR2DL4 & -0.006 & $8.88 \mathrm{e}-01$ & -0.011 & $8.08 \mathrm{e}-01$ \\
\hline & KIR3DL1 & 0.011 & $8 \mathrm{e}-01$ & 0.003 & $9.48 \mathrm{e}-01$ \\
\hline & KIR3DL2 & 0.055 & $2.16 \mathrm{e}-01$ & 0.055 & $2.26 \mathrm{e}-01$ \\
\hline & KIR3DL3 & 0.002 & $9.67 \mathrm{e}-01$ & 0.003 & $9.44 \mathrm{e}-01$ \\
\hline & KIR2DS4 & 0.007 & $8.75 e-01$ & 0.007 & $8.75 e-01$ \\
\hline \multirow{7}{*}{$\begin{array}{l}\text { Dendritic } \\
\text { cell }\end{array}$} & HLA-DPB1 & 0.397 & $0 \mathrm{e}+00$ & 0.399 & $4.01 \mathrm{e}-20$ \\
\hline & HLA-DQB1 & 0.38 & $0 \mathrm{e}+00$ & 0.38 & $3.53 e-18$ \\
\hline & HLA-DRA & 0.404 & $0 \mathrm{e}+00$ & 0.403 & $1.57 \mathrm{e}-20$ \\
\hline & HLA-DPA1 & 0.399 & $0 \mathrm{e}+00$ & 0.399 & $4.49 \mathrm{e}-20$ \\
\hline & BDCA-1 (CD1C) & 0.362 & $3.29 \mathrm{e}-17$ & 0.37 & $2.94 \mathrm{e} 17$ \\
\hline & BDCA-4 (NRP1) & -0.021 & $6.41 \mathrm{e}-01$ & -0.029 & $5.28 \mathrm{e}-01$ \\
\hline & CD11c (ITGAX) & 0.268 & $9.11 \mathrm{e}-10$ & 0.263 & $3.51 \mathrm{e}-09$ \\
\hline \multirow[t]{5}{*}{ Th1 } & T-bet (TBX21) & 0.119 & $7.18 \mathrm{e}-03$ & 0.116 & $1.03-02$ \\
\hline & STAT4 & 0.373 & $0 \mathrm{e}+00$ & 0.367 & $5.35 e-17$ \\
\hline & STAT1 & 0.379 & $8.53 e-19$ & 0.383 & $1.56 \mathrm{e}-18$ \\
\hline & IFN-g (IFNG) & 0.211 & $1.48 \mathrm{e}-06$ & 0.209 & $3.08 \mathrm{e}-06$ \\
\hline & TNF-a (TNF) & 0.243 & $2.9 \mathrm{e}-08$ & 0.241 & 7.47e-08 \\
\hline \multirow[t]{4}{*}{ Th2 } & GATA3 & 0.09 & $4.24 \mathrm{e}-02$ & 0.09 & $3.39 \mathrm{e}-03$ \\
\hline & STAT6 & 0.352 & $2.94 \mathrm{e}-16$ & 0.347 & $3.09 \mathrm{e}-15$ \\
\hline & STAT5A & 0.333 & $1.25 \mathrm{e}-14$ & 0.329 & $8.92 \mathrm{e}-14$ \\
\hline & IL13 & 0.092 & $3.84 \mathrm{e}-02$ & 0.085 & $6.07 \mathrm{e}-02$ \\
\hline Tfh & BCL6 & -0.016 & $7.2 \mathrm{e}-01$ & -0.032 & 4.76-e-01 \\
\hline \multirow[t]{2}{*}{ Th17 } & STAT3 & 0.254 & $6.78 \mathrm{e}-09$ & 0.249 & $2.58 \mathrm{e}-08$ \\
\hline & IL17A & 0.09 & $4.33 \mathrm{e}-02$ & 0.098 & $2.96 \mathrm{e}-02$ \\
\hline Treg & FOXP3 & 0.328 & $4.11 \mathrm{e}-14$ & 0.339 & $1.46 \mathrm{e}-14$ \\
\hline & CCR8 & 0.26 & $2.64 \mathrm{e}-09$ & 0.268 & $1.80 \mathrm{e}-09$ \\
\hline & STAT5B & 0.074 & $9.65 \mathrm{e}-02$ & 0.067 & $1.40 \mathrm{e}-01$ \\
\hline & TGFb (TGFB1) & 0.226 & $2.54 \mathrm{e}-07$ & 0.219 & $1.01 \mathrm{e}-06$ \\
\hline $\mathrm{T}$ cell & PD-1 (PDCD1) & 0.058 & $1.89 \mathrm{e}-01$ & 0.064 & $1.60 \mathrm{e}-01$ \\
\hline exhaustion & CTLA4 & 0.275 & $2.76 \mathrm{e}-10$ & 0.276 & $5.31 \mathrm{e}-10$ \\
\hline & LAG3 & 0.211 & $1.59 \mathrm{e}-06$ & 0.209 & $3.24 \mathrm{e}-06$ \\
\hline & TIM-3 (HAVCR2) & 0.272 & $5.12 \mathrm{e}-10$ & 0.269 & $1.46 \mathrm{e}-09$ \\
\hline & GZMB & 0.143 & $1.23 \mathrm{e}-03$ & 0.14 & $1.91 \mathrm{e}-03$ \\
\hline
\end{tabular}

We first examined the expression of ADORA1 in PTC according to the data from TCGA database and the Oncomine database. The results showed that ADORA1 mRNA levels were up-regulated in PTC compared to paracancerous tissues. And there were significant correlations between ADORA1 expression and the clinical stage, metastasis and survival analyses of PTC patients. To verify the bioinformatics predictions regarding the ADORA1 gene, we performed immunohistochemistry experiments on PTC tissue microarrays containing 58 pairs of tissues. As shown by the immunohistochemistry results, ADORA1 was overexpressed in PTC and had positive correlations with pathological stage and lymph node metastasis in Shanghai cohort (Table 1). Dysregulation of ADORA1 overexpression can promote the development of multiple solid tumors. Previous reports pointed out that ADORA1 antagonists could effectively reduce breast cancer cell line Mcf-7 cell proliferation [15]. And ADORA1 could regulate the apoptotic effect of breast cancer cells by regulating p53 expression [36]. In our study, the AUC of ADORA1 in TCGA cohort was 0.9408 and the AUC of ADORA1 in Shanghai cohort was 0.9064. Thus, ADORA1 can be considered as a potential diagnostic indicator of PTC and may play a crucial role in PTC.

Next, we queried the genes significantly correlated with ADORA1 and their functions in the PTC. GO term annotations showed that the fuctions of ADORA1 correlated genes mainly were involved in adaptive immune response, immune response-regulation signaling pathway, regulation of leukocyte activation, granulocyte activation, cilium organization, mitochondrial gene expression, response to virus, microtubule-based movement and generation of precursor metabolites and energy. And the KEGG pathway data indicated that ADORA1 and its related genes were primarily associated with cell adhesion molecules(CAMs), natural killer cell-mediated cytotoxicity, Th17 cell differentiation, epstein-barr virus infection, bacterial invasion of epithelial cells, phagosome, influenza A, complement and coagulation cascades and valine, leucine and isoleucine degradation. These results all suggested that ADORA1 might have a modulatory effect on cancer progression, especially immunomodulation. Besides, our data showed that ADORA1 could activate EMT, Hormone ER, PI3K/AKT, RAS/MAPK, TSC/mTOR pathways and inhibit RTK, Hormone AR, DNA Damage Response, Cell Cycle, Apoptosis pathways to exert regulatory effects on the cancer process. Sitkovsky et al pointed out that adenosine receptors on the surface of immune cells, especially $\mathrm{T}$ lymphocytes, could be activated rapidly and abundantly to regulate immune function when under oxygen-deficient environment [37]. Moreover, ADORA1 exerted a modulatory effect on cancer cell proliferation and differentiation by manipulating the MAPK signaling pathway [38]. Consequently, the functions of ADORA1 and related genes were 
primarily involved in tumor immunomodulation and cancer-related pathways, suggesting that ADORA1 may mediate the tumorigenesis and progression of PTC.

Genomic instability and mutations greatly increase the chances of tumorigenesis, while kinases and their associated regulators help stabilize and repair genomic DNA that has been mutated [39]. We found significant ADORA1 gene CNV alterations in PTC patients with clinical stage III-IV and extrathyroidal extension. This suggested that ADORA1 gene $\mathrm{CNV}$ changes can contribute to the generation of PTC. To further explore the possible mechanism in PTC, we analyzed the kinase, miRNA or transcription factor of ADORA1 using the
LinkedOmics database. STK11, a kinase involved in the regulation of ADORA1, was aberrantly expressed in various tumors and played a role in promoting cancer cell adhesion and angiogenesis [40]. Besides, LKB1 has been used for immunotherapy [41]. In this study, regulators of ADORA1 networks were mainly riched in adaptive immune response, immune response-regulation signaling pathway, regulation of leukocyte activation, granulocyte activation, microtubule-based movement, cell adhesion molecules (CAMs), natural killer cell-mediated cytotoxicity, Th17 cell differentiation and several cancer-related pathways. These suggested that ADORA1 can exert a pro-tumorigenic effect on PTC by modulating these regulators.

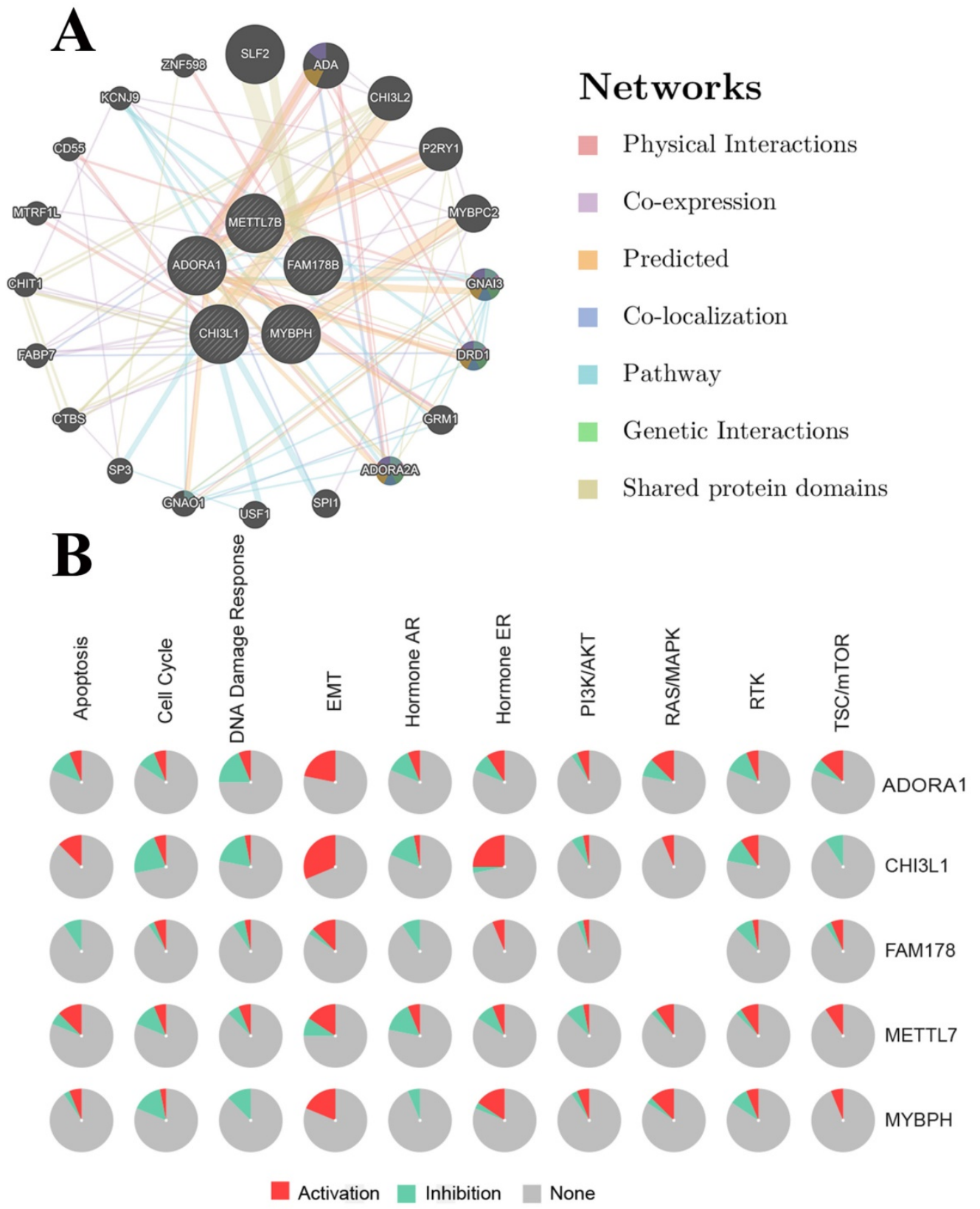

Figure 10. PPI network of ADORAl (A) and the role of ADORAl in the cancer related pathways (B). 


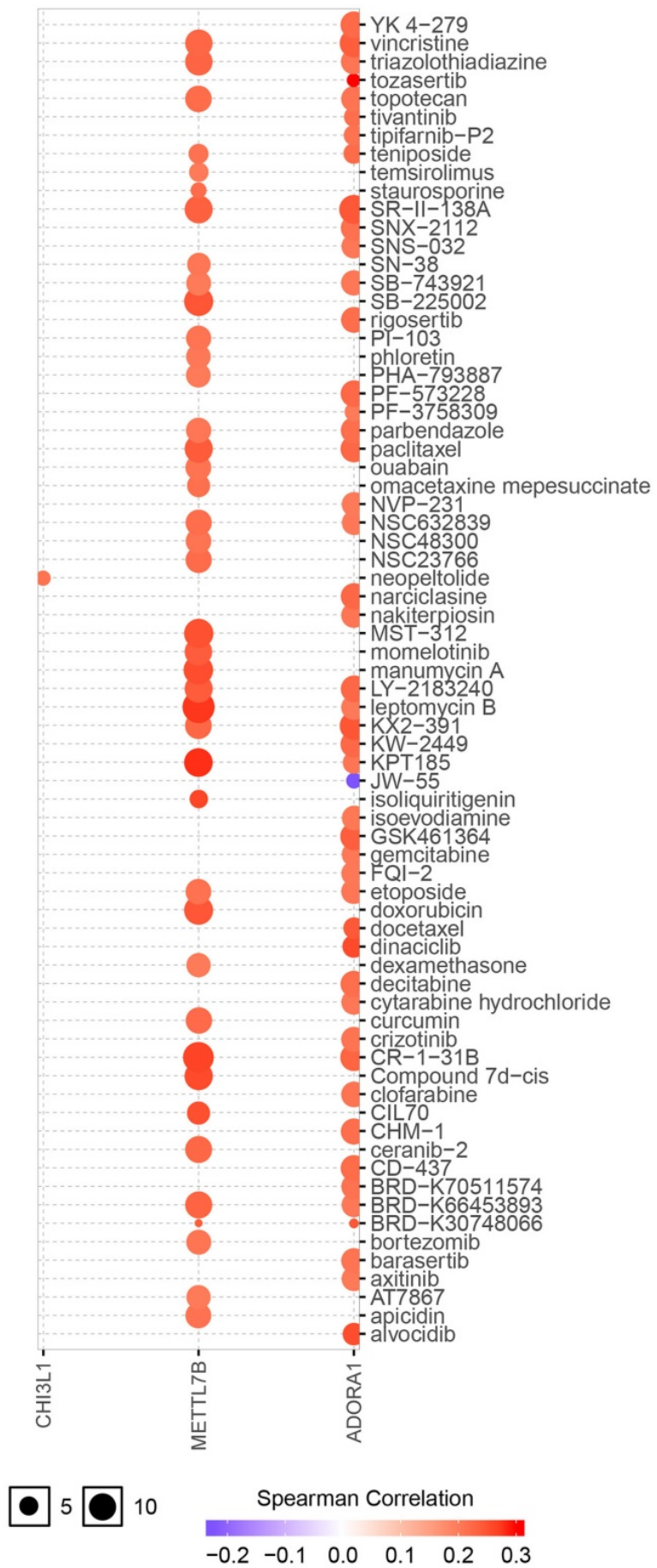

Figure 11. Drug susceptibility analysis of hub genes according to Therapeutics Response Portal (CTRP) data. A positive correlation between gene expression and drug indicates that the gene has an antagonistic effect on the drug.

Previous study proved that silencing ADORA1 expression significantly increased tumor PD-L1 levels and inhibited T-cell-mediated cytotoxicity in human melanoma cell lines [11]. And ADORA1 exerted immunoprotective effects on the organism by activating the immune response. Sitkovsky indicated that ADORA1 on endothelial cells and neutrophils could be activated by adenosine to enhance the ability of cell chemotaxis and release reactive oxygen species to participate in the immune response [37]. And there was growing evidence that ADORA1 was strongly associated with immunity. Here, the relationships between ADORA1 expression levels and immune cells and immune markers were analyzed comprehensively. And remarkable correlations were found between ADORA1 expression and the expression of CD2, CD115, IRF5, CD11b, HLA-DPB1, HLA-DQB1, HLA-DRA, HLA-DPA1, BDCA-1, STAT4, STAT6, STAT5A and FOXP3. In addition, the relationships between different ADORA1 expression and immune cells in PTC patients were analyzed using the EPIC application, and we found that different levels of ADORA1 expression was associated with B cells, CAFs, CD8 T cells, endothelial cells and macrophages. Finally, we found that high expression of ADORA1 was sensitive to JW-55 drug. Thus, ADORA1 may play an essential role in the immune microenvironment of PTC, and ADORA1 may be an important immune checkpoint in PTC.

To summarize, ADORA1 was overexpressed in PTC and can be a diagnostic and a prognostic factor. The functions of ADORA1 and associated genes were mainly involved in adaptive immune response, immune response-regulation signaling pathway, regulation of leukocyte activation, cell adhesion molecules (CAMs), natural killer cell-mediated cytotoxicity, Th17 cell differentiation and cancerrelated pathways. ADORA1 expression was significantly correlated with immune cells and immune markers. These results all suggest that ADORA1 may play an oncogenic role in PTC.

\section{Supplementary Material}

Supplementary figures.

http://www.jcancer.org/v12p3997s1.pdf

\section{Acknowledgements}

\section{Funding}

Hebei North University 2019 Basic Research Business Expenses Project (NO. JYT2019015); Hebei Provincial Department of Finance Specialist Capacity Building and Specialist Leadersa Program, China (NO. 361009); Hebei Provincial Natural Science Foundation Project (NO. H201840505); Science and Technology Plan Project of Zhangjiakou City (NO. 1911019D).

Grant sponsor: Hebei North University 2019 Basic Research Business Expenses Project.

Grant number: JYT2019015.

\section{Author Contributions}

Jing-Fang $\mathrm{Wu}$ designed the study. This article was written by $\mathrm{Xu}$ Lin. Geng Zhang and Zhi-Yong 
Wang conducted the statistical analysis. Gang Xue was in charge of directing. All author-approved final drafts.

\section{Competing Interests}

The authors have declared that no competing interest exists.

\section{References}

[1] Cancer Genome Atlas Research N. Integrated genomic characterization of papillary thyroid carcinoma. Cell. 2014; 159(3):676-90.

[2] La Vecchia C, Malvezzi M, Bosetti C, et al. Thyroid cancer mortality and incidence: a global overview. Int J Cancer. 2015; 136(9):2187-95.

[3] Pourseirafi S, Shishehgar M, Ashraf MJ, et al. Papillary Carcinoma of Thyroid with Nasal Cavity Metastases: A Case Report. Iran J Med Sci. 2018; 43(1):90-3.

[4] Rosenbaum MA, Mchenry CR. Contemporary management of papillary carcinoma of the thyroid gland. Expert Rev Anticancer Ther. 2009; 9(3):317-29.

[5] Ullmann TM, Gray KD, Moore MD, et al. Current controversies and future directions in the diagnosis and management of differentiated thyroid cancers. Gland Surg. 2018; 7(5):473-86.

[6] Duman BB, Kara OI, Uguz A, et al. Evaluation of PTEN, PI3K, MTOR, and KRAS expression and their clinical and prognostic relevance to differentiated thyroid carcinoma. Contemp Oncol (Pozn). 2014; 18(4):234-40.

[7] Zhao Y, Zhong L, Yi H. A review on the mechanism of iodide metabolic dysfunction in differentiated thyroid cancer. Mol Cell Endocrinol. 2019; 479:71-7.

[8] Vigneri R, Malandrino P, Vigneri P. The changing epidemiology of thyroid cancer: why is incidence increasing? Curr Opin Oncol. 2015; 27(1):1-7.

[9] Kim K, Jeon S, Kim TM, et al. Immune Gene Signature Delineates a Subclass of Papillary Thyroid Cancer with Unfavorable Clinical Outcomes. Cancers (Basel). 2018; 10(12):1-18.

[10] Gao ZG, Jacobson KA. A2B Adenosine Receptor and Cancer. Int J Mol Sci. 2019; 20(20):5139-57.

[11] Liu H, Kuang X, Zhang Y, et al. ADORA1 Inhibition Promotes Tumor Immune Evasion by Regulating the ATF3-PD-L1 Axis. Cancer Cell. 2020; 37(3):324-39 e8.

[12] Kazemi MH, Raoofi MS, Hojjat-Farsangi M, et al. Adenosine and adenosine receptors in the immunopathogenesis and treatment of cancer. J Cell Physiol. 2018; 233(3):2032-57.

[13] Gessi S, Merighi S, Varani K, et al. Adenosine receptors in colon carcinoma tissues and colon tumoral cell lines: focus on the A(3) adenosine subtype. J Cell Physiol. 2007; 211(3):826-36.

[14] Zhou Y, Tong L, Chu X, et al. The Adenosine A1 Receptor Antagonist DPCPX Inhibits Tumor Progression via the ERK/JNK Pathway in Renal Cell Carcinoma. Cell Physiol Biochem. 2017; 43(2):733-42.

[15] Lin Z, Yin P, Reierstad S, et al. Adenosine A1 receptor, a target and regulator of estrogen receptoralpha action, mediates the proliferative effects of estradiol in breast cancer. Oncogene. 2010; 29(8):1114-22.

[16] Daniele S, Zappelli E, Natali L,et al. Modulation of A1 and A2B adenosine receptor activity: a new strategy to sensitise glioblastoma stem cells to chemotherapy. Cell Death Dis. 2014; 5:e1539-54.

[17] Gessi S, Varani K, Merighi S, et al. Pharmacological and biochemical characterization of A3 adenosine receptors in Jurkat $\mathrm{T}$ cells. Br J Pharmacol. 2001; 134(1):116-26.

[18] Gong J, Chehrazi-Raffle A, Reddi S,et al. Development of PD-1 and PD-L1 inhibitors as a form of cancer immunotherapy: a comprehensive review of registration trials and future considerations. J Immunother Cancer. 2018; 6(1):8-26.

[19] Ni JM, Ni AP. Landscape of PD-1/PD-L1 Regulation and Targeted Immunotherapy. Chin Med Sci J. 2018; 33(3):174-82

[20] Shen X, Zhao B. Efficacy of PD-1 or PD-L1 inhibitors and PD-L1 expression status in cancer: meta-analysis. BMJ. 2018; 362:k3529-38

[21] Gao J, Aksoy BA, Dogrusoz U, et al. Integrative analysis of complex cancer genomics and clinical profiles using the cBioPortal. Sci Signal. 2013; 6(269):pl1-34.

[22] Chandrashekar DS, Bashel B, Balasubramanya SAH, et al. UALCAN: A Portal for Facilitating Tumor Subgroup Gene Expression and Survival Analyses. Neoplasia. 2017; 19(8):649-58.

[23] Rhodes DR, Yu J, Shanker K, et al. ONCOMINE: a cancer microarray database and integrated data-mining platform. Neoplasia. 2004; 6(1):1-6.

[24] Gyorffy B, Lanczky A, Eklund AC, et al. An online survival analysis tool to rapidly assess the effect of 22,277 genes on breast cancer prognosis using microarray data of 1,809 patients. Breast Cancer Res Treat. 2010; 123(3):725-31.

[25] Vasaikar SV, Straub P, Wang J,et al. LinkedOmics: analyzing multi-omics data within and across 32 cancer types. Nucleic Acids Res. 2018; 46(D1):D956-D63.

[26] Lin X, Zhang H, Dai J, et al. TFF3 Contributes to Epithelial-Mesenchymal Transition (EMT) in Papillary Thyroid Carcinoma Cells via the MAPK/ERK Signaling Pathway. J Cancer. 2018; 9(23):4430-9.
[27] Remmele W, Stegner HE. [Recommendation for uniform definition of an immunoreactive score (IRS) for immunohistochemical estrogen receptor detection (ER-ICA) in breast cancer tissue]. Pathologe. 1987; 8(3):138-40.

[28] Warde-Farley D, Donaldson SL, Comes O, et al. The GeneMANIA prediction server: biological network integration for gene prioritization and predicting gene function. Nucleic Acids Res. 2010; 38 (Web Server issue):W214-20.

[29] Liu CJ, Hu FF, Xia MX, et al. GSCALite: a web server for gene set cancer analysis. Bioinformatics. 2018; 34(21):3771-2.

[30] Li T, Fan J, Wang B, et al. TIMER: A Web Server for Comprehensive Analysis of Tumor-Infiltrating Immune Cells. Cancer Res. 2017; 77(21):e108-e10.

[31] $\mathrm{Ru} \mathrm{B}$, Wong CN, Tong $\mathrm{Y}$, et al. TISIDB: an integrated repository portal for tumor-immune system interactions. Bioinformatics. 2019; 35(20):4200-2.

[32] Racle J, De Jonge K, Baumgaertner P, et al. Simultaneous enumeration of cancer and immune cell types from bulk tumor gene expression data. Elife. 2017; 6: e26476-501.

[33] Cekic C, Linden J. Purinergic regulation of the immune system. Nat Rev Immunol. 2016; 16(3):177-92.

[34] Hasko G, Cronstein B. Regulation of inflammation by adenosine. Front Immunol. 2013; 4:85-93.

[35] Hasko G, Linden J, Cronstein B,et al. Adenosine receptors: therapeutic aspects for inflammatory and immune diseases. Nat Rev Drug Discov. 2008; 7(9):759-70

[36] Dastjerdi MN, Valiani A, Mardani M,et al. Adenosine A1 receptor modifies P53 expression and apoptosis in breast cancer cell line Mcf-7. Bratisl Lek Listy. 2016; 117(4):242-6.

[37] Sitkovsky M, Lukashev D. Regulation of immune cells by local-tissue oxygen tension: HIF1 alpha and adenosine receptors. Nat Rev Immunol. 2005; 5(9):712-21.

[38] Ciruela F, Albergaria C, Soriano A, et al. Adenosine receptors interacting proteins (ARIPs): Behind the biology of adenosine signaling. Biochim Biophys Acta. 2010; 1798(1):9-20.

[39] Helm JS, Rudel RA. Adverse outcome pathways for ionizing radiation and breast cancer involve direct and indirect DNA damage, oxidative stress, inflammation, genomic instability, and interaction with hormonal regulation of the breast. Arch Toxicol. 2020; 94(5):1511-49.

[40] Granado-Martinez P, Garcia-Ortega S, Gonzalez-Sanchez E, et al. STK11 (LKB1) missense somatic mutant isoforms promote tumor growth, motility and inflammation. Commun Biol. 2020; 3(1):366-80.

[41] Wang H, Guo J, Shang X, t al. Less immune cell infiltration and worse prognosis after immunotherapy for patients with lung adenocarcinoma who harbored STK11 mutation. Int Immunopharmacol. 2020; 84:106574-9. 